\title{
Reversible Watermarking using Histogram Shifting and Pixel Differences with Inverse S-scan order and Local Prediction
}

\author{
Naseera P. A \\ Computer Science and Engineering, ICET, Muvattupuzha, India
}

\begin{abstract}
The Reversible Watermarking enables the embedding of Watermarks in a host image without any loss of host content, which is proposed for image authentication that if the watermarked image is deemed authentic, it can revert in to the exact copy of the original image before the embedding occurred. This paper convey the improved histogram-based reversible watermarking scheme based pixel differences with local prediction. Before performing the embedding process flip the host image, For each pixel, calculate the difference between them. And take the difference value for finding peak value of the histogramand hide the watermarkin the flipped image's histogram peaks. This will perform high security in data hiding. The revers process is performed at the receiver.
\end{abstract}

Keywords: Reversible watermarking, difference expansion, Histogram Shifting, Image Flipping, Inverse S-scan order.

\section{Introduction}

In the recent years, the development of multimedia technologies and internet has provided the consumers theaccess to multimedia data. Hence the data may be tampered orattacked during transmission. So it is essential to protect thedigital data in applications like military or medicalapplications [4]. A possible solution to prevent such forgery isdata hiding techniques. Data hiding refers to hide data within a digital media. Mediacan be anything like audio, image and video. Hiding is doneby modifying the contents of the digital media. Hiding processis done in such a way that modification of pixel values shouldbe undetectable to the viewers. Image where the hiding isdone is known as the cover media. Image after data hiding isknown as watermarked image.In most hiding techniques, the host image is distortedpermanently and thus it cannot be recovered back completelyfrom the marked content. However, in various applicationslike military and medical applications, degradation of the overmedia is not allowed. For medical applications, even the slightchanges in the image are intolerable. So it is essential tointroduce the data hiding in such a way that it is reversible andquality degradation after embedding is lowered. Suchreversible data hiding techniques are also known as distortionless or lossless data hiding techniques. Reversible data hidingtechniques hides information within the digital media in sucha way that only the authorized person could decode the hiddendata and restore back to the original state.

\subsection{Histogram Modification}

In [9], Ni et al. introduced a reversible data hiding scheme based on histogram modification.Itwill describe briefly in this section. The histogram modification technique involves generating histogram and finding the peak point and the zero point and shifting histogram bins to embed message bits.

For a given host image, it will generate its histogram and find a peak point and a zero point. A peak point corresponds to the grayscale value which the maximum number of pixels in the given image assumes. On the contrary, a zero point corresponds to the grayscale value which no pixel in the given image assumes. By considering Histogram of any image and, Let $P$ be the value of peak point and $Z$ be the value of zero point. The range of the histogram, $[P+1, Z-1]$, is shifted to the right-hand side by 1 to leave the zero point at $P+1$. Once a pixel with value $P$ is encountered, if the message bit is " 1 ," increase the pixel value by 1 . Otherwise, no modification is needed. Here the number of message bits that can be embedded into an image equals to the number of pixels which are associated with the peak point. The data extraction is actually the reverse process of data hiding. When a pixel with value $P+1$ is met, message bit " 1 " is extracted and the pixel value reduces to $P$. When a pixel with value $P$ is met, message bit " 0 " is extracted. After all message bits have been extracted, shift the range of the histogram, $[P+2, Z]$, to the left-hand side by 1 . Note that zero point defined above may not exist in some image histograms. In this regard, a minimum point that is defined as the grayscale value which the minimum number of pixels in the given image assumes is often used in place of the zero point. However, the grayscale value and coordinate of the pixel that is associated with the minimum point need to be recorded as overhead bookkeeping information. Therefore, if the required payload is larger than the actual hiding capacity that is referred to as pure payload, more pairs of peak and minimum points need to be used.

The algorithm steps are:

1) Scan the cover image and construct its histogram

2) Thegray value for which the histogram is highest is denoted the peak point $\mathrm{P}$, and the gray value for which the histogram is lowest is denoted by the minimum point Z

3) If $\mathrm{Hi}(Z)=0$, then $Z$ is called a zero point. For simplicity, we assume $\mathrm{P}<\mathrm{Z}$

4) Scan the image and record the positions of those pixel values to $Z$

5) Shift the histogram $\operatorname{Hi}(x), x \in(P, Z)$ to the right to vacate the histogram bin at $\mathrm{P}+1$

6) Extract a data bit $\mathrm{s}$ from secret data S. Scan the image oncemore 


\section{International Journal of Science and Research (IJSR) \\ ISSN (Online): 2319-7064}

Index Copernicus Value (2013): 6.14 | Impact Factor (2014): 5.611

7) If the scanned pixel value is $P$ and the data bit to be embedded is 1 , then set the pixel value to $\mathrm{P}+1$

8) If the data bit to be embedded is 0 , no change has to be done on the scanned pixel [1]

The similar process is required to be followed for the lossless recovery of the Original Image and the watermark.

\section{Related Work}

Most of the data hiding techniques are not reversible completely. The well-known LSB technique is not completelyreversible due to bit replacement without "memory".Reversible data hiding techniques are mainly classified intothree main categories, first is based on data compression [2], [5], [6], second based on pixel value difference expansion [3], [10] and third based on histogram shifting [8], [13]. All theseearliest technique is based on LSB replacement. In Datacompression technique, the data to be embedded as well asrelated information's of the image used for data recovery iscompressed. Compressed data is embedded directly into thecover media using LSB replacement. Celik et al. [6] proposeda data hiding technique in which each image pixel is quantizedusing L-level scalar technique. The residues yielded afterquantization is compressed using a lossless compressionalgorithm called CALIC. Compressed residues along with theto-be embedded bits are embedded into the quantised imageusing LSB replacement. Distortions introduced on thewatermarked image by this method are comparatively high.Technique based on pixel value difference expansiondiscovers the redundancy of pixel values in the images.Arithmetic operations are performed on the pixel pair's inorder to explore the space for data embedding. Whether thepixel values can be expanded or not is indicated by thelocation map. This technique achieves higher embeddingcapacity and keeps the distortion low. Tian et al [3]]proposedthe data hiding technique in which data bits are embedded bycomputing the difference between the pixel pairs. Locationmap which is essential for image restoration is embeddedalong with the data bits. Coltuc et al[11]proposed a datahiding technique in which embedding of data bits is done bytaking the RCM transform of the image. Reversible contrastmapping is an integer transform which is invertible even ifsome LSB bits are lost. Data bits are embedded in the LSBbits of such transformed pixel pairs. Complexity of thetechnique based on pixel value difference expansion is less, thus hacking becomes easier.Histogram based data hiding technique embeds the data inthe cover media by shifting the histogram of the image. Histogram technique finds peak or zero points in thehistogram and data embedding is done by shifting these peak and zero points. This technique yields higher data hidingcapacity with low distortion. Histogram based reversible datahiding method was introduced by $\mathrm{Ni}$ et al. in [8], wheremessage is embedded within the histogram. Embedding isdone by shifting the peak and zero points of the histogram. Also histogram shifting technique prevents overflow and underflow problem. Overflow is the condition that the greyvalue exceeds above 255. Underflow is the condition that thegreyvalue falls below 0 .Embedding based on histogram modification has beenpresented in [7], [8], [12].
The Proposed approach adopts the histogram shifting technique; this will improve the hiding capacity and high security for watermarking.

\section{Proposed Approach}

The proposed scheme improves hiding capacity and high security for the watermark. It is very difficult to find watermarked bits by an unauthorized person. Thus the new method describes an efficient extension of the histogram modification technique by considering the difference between adjacent pixels instead of simple pixel value. The distribution of pixel difference has a prominent maximum since image neighbor pixels are strongly correlated. Hence, there are a lot of candidates for embedding data; Characteristics of the pixel difference are exploited to achieve large hiding capacity while keeping the embedding distortion low. Inverse $\mathrm{S}$ order scanning and image flipping provide high security in embedded data. Histogramshifting technique also prevents overflow and underflow.

\subsection{Calculation of pixel differences and Histogram modification}

The reversible data hiding scheme is designed as follows.

1) Read the cover image and perform inverse $S$-scan order.

(Scan the even rows of the image matrix.)

2) Then flip the scanned image. And save that value in the even position of image matrix.

3) Calculate the pixel difference $d_{i}$ between pixels $x_{i-1}$ and $x_{i}$ by

$$
d_{i}=\left\{\begin{array}{c}
x_{i}, \quad \text { if } i=0, \\
\left|x_{i-1}-x_{i}\right|, \text { otherwise }
\end{array}\right\}
$$

4) Determine the peak point $P$ from the pixel differences.

5) If $d_{i}>P$, shift $x_{i}$ by 1 unit:

$$
y_{i}=\left\{\begin{array}{c}
x_{i}, \quad \text { if } i=0 \text { or } d_{i}<P \\
x_{i}+1, \text { if } d_{i}>P \text { and } x_{i} \geq x_{i-1} \\
x_{i}-1, \text { if } d_{i}>P \text { and } x_{i}<x_{i-1}
\end{array}\right\}
$$

Where $y_{i}$ is the watermarked value of pixel $i$.

6) If $d_{i}=P$, modify $x_{i}$ according to the message bit:

$$
y_{i}=\left\{\begin{array}{l}
x_{i}+b, \text { if } d_{i}=P \text { and } x_{i} \geq x_{i-1} \\
x_{i}-b, \text { if } d_{i}=P \text { and } x_{i}<x_{i-1}
\end{array}\right\}
$$

Where $y_{i}$ is the watermarked value of pixel $i$.

7) Construct the watermarked bits according to the pixels $y_{i}$.

At the receiving end, the recipient extracts message bits from the watermarked image by scanning the image in the same order as during the embedding.

The message bit $b$ can be extracted by

$$
b=\left\{\begin{array}{c}
0, \text { if }\left|y_{i}-x_{i-1}\right|=P, \\
1, \text { if }\left|y_{i}-x_{i-1}\right|=P+1,
\end{array}\right\}
$$

where $x_{i-1}$ denotes the restored value of $y_{i-1}$. Then the original pixel value of $x_{i}$ can be restored by

$$
y_{i}=\left\{\begin{array}{c}
y_{i}+1, \text { if }\left|y_{i}-x_{i-1}\right|>P \text { and } y_{i}<x_{i-1}, \\
y_{i}-1, \text { if }\left|y_{i}-x_{i-1}\right|>P \text { and } y_{i}>x_{i-1} \\
y_{i}, \quad \text { otherwise }
\end{array}\right\}
$$




\section{International Journal of Science and Research (IJSR) \\ ISSN (Online): 2319-7064}

Index Copernicus Value (2013): 6.14 | Impact Factor (2014): 5.611

Thus, the exact copy of the original host image is obtained.

\section{Conclusion}

This paper, proposes an efficient extension of histogram modification by considering the difference between adjacent pixels instead of simple pixel value. The distribution of pixel difference has a prominent maximum since neighbor pixels are strongly correlated. The watermark bits are embedded on the histogram peaks, so the embedding capacity is improved. Inverse S-scan order and image flipping provides high security for embedded data.

\section{Acknowledgment}

The Author would like to thank Femithamol. A. M. Assistant Professor, Department of Information Technology, Ilahia College of Engineering and Technology, Muvattupuzha for her moral and technical support.

\section{References}

[1] Celik, M.U., Sharma, G., Tekalp, A.M., and Saber, E.:,Reversible data hidinge. Proc. ICIP, 2002, vol. 2, pp. 157-160

[2] J. Fridrich, M. Goljan, and R. Du, "Lossless data embedding-new paradigmin digital watermarking," Eur. Assoc. Signal Process. J. Appl. Signal Process.,vol. 2002, no. 2, pp. 185-196, Feb. 2002.

[3] J. Tian, "Reversible data embedding using a difference expansion," IEEETrans. Circuits Syst. Video Technol., vol. 13, no. 8, pp. 890-896, Aug. 2003.

[4] R.Norcen, M.Podesser, A.Pommer, H.Schmidt, and A.Uhl, "ConfidentialStorage and Transmission of Medical Image Data" Computers in Biology andMedicine 33, pp.277-292, 2003.

[5] T. Kalker and F. M. J. Willems, "Capacity bounds and constructions forreversible data hiding," Security Watermarking Multimedia Contents V, vol.5020, pp. 604-611, Jun. 2003.

[6] M. U. Celik, G. Sharma, A. M. Tekalp, and E. Saber, "Losslessgeneralized-LSB data embedding," IEEE Trans. Image Process., vol. 14, no.2, pp. 253-266, Feb. 2005.

[7] L. Kamstra and H. J. A. M. Heijmans, "Reversible data embedding intoimages using wavelet techniques and sorting," IEEE Trans. Image Process.,vol. 14, no. 12, pp. 2082-2090, Dec. 2005.

[8] S. K. Lee, Y. H. Suh, and Y. S. Ho, "Reversible image authenticationbased on watermarking," in Proc. IEEE Int. Conf. Multimedia Expo, Toronto,ON, Canada, Jul. 2006, pp. 1321-1324.

[9] Z. Ni, Y. Q. Shi, N. Ansari, and W. Su, "Reversible data hiding," IEEE Transactions on Circuits and Systems for Video Technology, vol. 16, no. 3, pp. 354-362, Mar. 2006.

[10] D. M. Thodi and J. J. Rodriguez, "Expansion embedding techniques forreversible watermarking," IEEE Trans. Image Process., vol. 16, no. 3, pp.721-730, Mar. 2007

[11]D. Coltuc and J. M. Chassery, "Very fast watermarking by reversiblecontrast mapping," IEEE Signal Process. Lett., vol. 14, no. 4, pp. 255-258,Apr.2007
[12] M. Fallahpour and M. H. Sedaaghi, " High capacity lossless data hidingbased on histogram modification," IEICE Electron. Exp., vol. 4, no. 7, pp.205-210, Apr. 2007.

[13] W. Hong, T. S. Chen, and C. W. Shiu, "Reversible data hiding for highquality images using modification of prediction errors," J. Syst. Softw., vol. 82,no. 11, pp. 1833-1842, Nov. 2009.

\section{Author Profile}

Naseera P.A received the Bachelor of Technology degree in Information Technology from Mahatma Gandhi University, Kerala. She is currently doing Master of Technology degree in Computer Science and Engineering with Specialization in Information Systems from Mahatma Gandhi University, Kerala. 